\title{
Uncovering the Mechanism for Electron-beam Manipulation of Dopants in Silicon
}

Alexander Markevich ${ }^{1}$, Bethany Hudak ${ }^{2}$, Andrew Lupini ${ }^{3}$ and Toma Susi ${ }^{1}$

${ }^{1}$ University of Vienna, Vienna, Wien, Austria, ${ }^{2}$ US Naval Research Laboratory, Washington, District of Columbia, United States, ${ }^{3}$ ORNL, Knoxville, Tennessee, United States

Covalently bound impurity atoms in crystal lattices can be manipulated via the elastic backscattering of probe electrons from nuclei using the atomically focused electron irradiation of a scanning transmission electron microscope [1]. Early work discovered the atomistic mechanism for manipulation in graphene: out-of-plane dynamics of a carbon atom neighboring an impurity, leading to what can be conceived of as a beam-controlled direct exchange process [2]. Most of the work in this newly emerging area has focused on lattice impurities in single-layer graphene, initially incidental silicon impurities $[3,4]$ but later also phosphorus dopants [5].

While manipulation in graphene and potentially other 2D materials - provided their ionization damage can be managed [6] - continues to be a rich area of study with several open questions and much room for development, the unique strength of electron-beam manipulation is its potential for accessing impurities within (thin) bulk crystals. Of particular technological importance, particularly for quantum information sciences, are impurity atoms in silicon. The first experimental demonstration of such manipulation was recently presented for bismuth dopants [7,8] introduced via chemical vapor deposition, but the precise mechanism remains uncertain.

We have recently applied our established ab initio modeling methodology [9] to address the manipulation of impurities within crystalline silicon, revealing a novel type of mechanism we call indirect exchange. In this process, a silicon neighbor of the impurity atom is knocked by an electron impact into a metastable interstitial configuration, with the impurity taking the lattice position originally occupied by the impacted silicon atom in the subsequent recombination. However, contrary to the earlier discovered process in graphene, the primary knocked-on atom does not end up as a neighbor of the impurity, but as a nextnearest neighbor, displacing another silicon atom during the dynamics.

We have modeled this process for all experimentally feasible group V dopant impurities, namely arsenic, antimony and bismuth, revealing both commonalities and differences between them that can be understood based on their bonding within the lattice. Importantly, the mechanism we have now uncovered is non-destructive and occurs without removing atoms from inside the crystal. Finally, we present new experimental data on the manipulation of antimony impurity atoms, further highlighting the great potential of electron-beam manipulation for engineering crystalline materials with atomic precision.

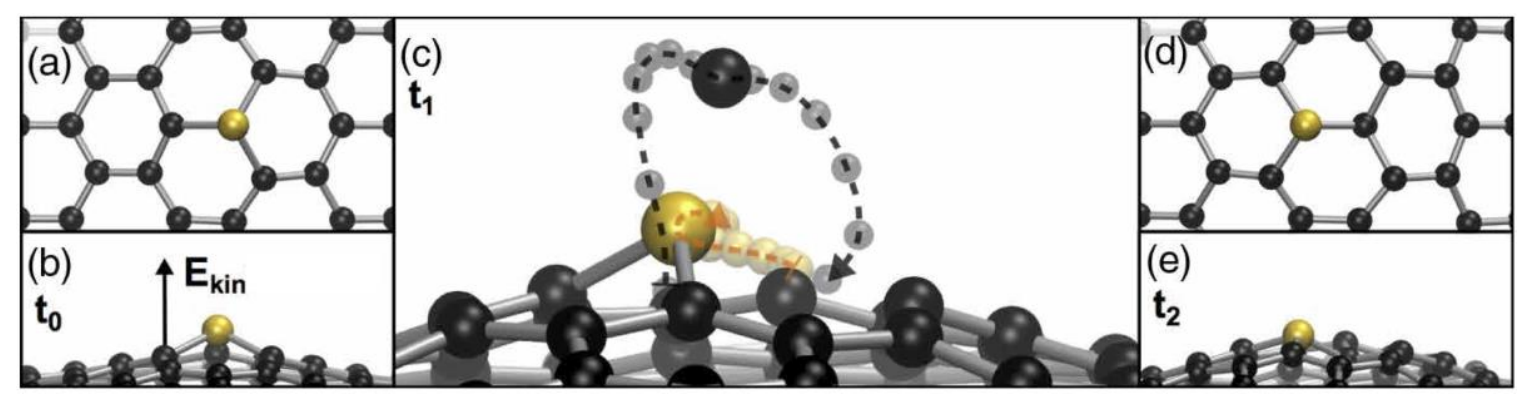


Figure 1. Direct exchange process in graphene. Molecular dynamics simulation of an out-of-plane dynamics of a $\mathrm{C}$ atom neighboring $\mathrm{Si}$ impurity in graphene after an electron impact delivering $15 \mathrm{eV}$. (a) Top view of the starting configuration at time $\mathrm{t} 0$. (b) Side view at $\mathrm{t} 0$, with the kinetic energy indicated on the impacted atom. (c) A snapshot at $\mathrm{t} 1$ ( $\sim 700$ time steps into the simulation), with the entire trajectories of the ejected $\mathrm{C}$ atom and the $\mathrm{Si}$ atom marked by semitransparent balls and dashed lines. (d) Top view near the end of the simulation at time $\mathrm{t} 2$ after $\sim 1400$ time steps. (e) Side view at time $\mathrm{t} 2$. Note that although the atomic motion has not ceased by this point, no further changes in the atomic configuration follow. From Ref. [2].

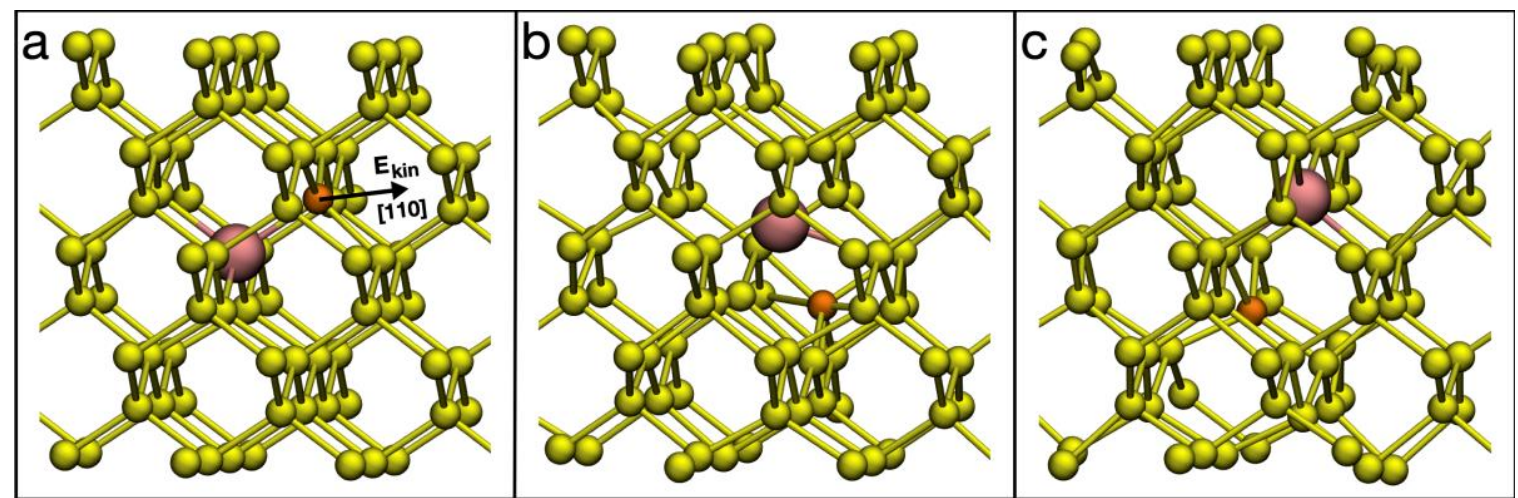

Figure 2. Indirect exchange process in bulk silicon. Snapshots of the molecular dynamics simulations showing (a) initial, (b) metastable interstitial and (c) final configurations of the Bi impurity atom, shown in pink color, in silicon after an electron impact delivering $15 \mathrm{eV}$ to its neighboring $\mathrm{Si}$ atom, shown in orange, in the [110] direction. Note that in the final configuration the knocked-on Si atom, shown in orange, became the second nearest neighbour.

\section{References}

[1] T. Susi et al., Ultramicroscopy 180 (2017) 163 [2] T. Susi et al., Physical Review Letters 113 (2014) 115501 [3] M. Tripathi et al., Nano Letters 18 (2018) 5319 [4] O. Dyck et al., Small 14 (2018) 1801771 [5] C. Su et al., Science Advances 5 (2019) eaav2252 [6] T. Susi, J.C. Meyer, J. Kotakoski, Nature Reviews Physics 1 (2019) 397 [7] S. Jesse et al., Nanotechnology 29 (2018) 255303 [8] B. Hudak et al., ACS Nano 12 (2018) 5873 [9] T. Susi et al., Nature Communications 7 (2016) 13040 